\title{
Reliability of Hip Migration Index in Children with Cerebral Palsy: The Classic and Modified Methods
}

\author{
Sun Mi Kim, M.D., Eun Geol Sim, M.D., Seong Gyu Lim, M.D., Eun Sook Park, M.D.
}

Department and Research Institute of Rehabilitation Medicine, Yonsei University College of Medicine, Seoul 120-752, Korea

Objective To determine reliability and clinical use of two methods of migration index (MI) in CP patients with or without hip dysplasia.

Method The materials included radiographs of 200 hips of children with cerebral palsy. Conventional anteroposterior radiographs of the pelvis were taken with the child in the supine position with standardized methods. Two rehabilitation doctors measured the migration index using two methods. In the classic method, the lateral margin of the acetabular roof was used as a landmark and in the modified method the lateral margin of the sourcil was used as a landmark. Each rater measured the migration index at three separate times with a time interval of at least one week. Intraclass correlation (ICC) was used to test the inter- and intra-rater reliability.

Results MI shows excellent intra-rater reliability in both the classic and modified methods, but the inter-rater reliability was higher in the classic method than in the modified method. When categorized according to the sourcil classification, inter-rater reliability was higher in the normal sourcil type and lower in the dysplastic sourcil types.

Conclusion Generally, the classic method showed higher reliability than the modified method, even though the reliability of the MI measurement was relatively high with both methods.

Key Words Cerebral palsy, Hip joint, Radiography, Migration index, Reliability

Received August 1, 2011; Accepted October 26, 2011

Corresponding author: Eun Sook Park

Department and Research Institute of Rehabilitation Medicine, Yonsei University College of Medicine, 134, Sinchon-dong, Seodaemun-gu, Seoul 120-752, Korea

Tel: +82-2-2228-3712, Fax: +82-2-363-2795, E-mail: pes1234@yuhs.ac (c) This is an open-access article distributed under the terms of the Creative Commons Attribution Non-Commercial License (http:// creativecommons.org/licenses/by-nc/3.0) which permits unrestricted noncommercial use, distribution, and reproduction in any medium, provided the original work is properly cited.

Copyright (C) 2012 by Korean Academy of Rehabilitation Medicine

\section{INTRODUCTION}

The incidence rate of hip displacement is the highest after equinus in musculoskeletal deformities in patients with cerebral palsy. If appropriate therapeutic intervention is not applied, hip displacement may develop into hip dislocation and cause postural and gait abnormalities, scoliosis, hip joint pains and problems of perineal hygiene. ${ }^{1,2}$ The subluxation or dislocation of the hip joint is one of the complications faced by children with cerebral palsy, in whom the incidence rate reaches $15 \%$ to $30 \%$ for all cerebral palsy patients . It is especially 
higher in patients with quadriplegic cerebral palsy.

Early therapeutic intervention is known to reduce the incidence rate of serious hip displacement in children with cerebral palsy. ${ }^{3}$ Thus, the early detection of hip displacement is critical to delay the progress of hip displacement. Various radiologic indexes have been used to detect hip displacement in children with cerebral palsy early. This is particularly true for the migration index, ${ }^{4}$ which is the most frequently used index to evaluate the risk of hip displacement and associated therapeutic effects.

In previous studies, intra-rater reliabilities were high, but inter-rater reliabilities showed different results. ${ }^{5-7}$

In most cases, the lateral edge of the acetabular roof is used as the datum point in the process where the migration index is measured. In the case of acetabular dysplasia, however, the lateral edge of the acetabular roof may be irregular in its coordinates, and therefore the measured values may be different among raters.

The sourcil is a shadow of dense osseous tissue that is observed at the weight-bearing surface of the acetabular roof (Fig. 1). In general, hip dysplasia can be evaluated by two methods, i.e., the classic method and the modified method. The former is to use the lateral edge of the acetabular roof as the datum point, and the latter is to use the lateral edge of the sourcil. In previous studies on the acetabular angles of patients who underwent operations for developmental hip displacement, it was reported that the modified method was more useful to evaluate hip dysplasia. $^{8-10}$

Also in children with cerebral palsy, the measured value may be different according to datum points.
Nevertheless, there has been no comparative study on the classic method and the modified method in relation to the migration index. This study analyzed intra-rater and inter-rater reliability not only in the classic method but in the modified method, as well as to study the clinical significance.

\section{MATERIALS AND METHODS}

\section{Subjects}

This study was conducted on patients with cerebral palsy who visited the rehabilitation department at this hospital and underwent plain radiographies on their hip joints (200 hip joints) between August 2009 and December 2009. The study group consisted of 66 boys and 34 girls, whose average age was $7.88 \pm 1.54$ years.

\section{Methods}

Subjects were assisted to take a supine position so their their pelvises could be parallel to each other and both their coxae could be in a neutral position while undergoing plain hip radiographies. ${ }^{11,12}$ This study was retrospectively conducted, and hip displacement was measured by two raters (A and B) using plain hip radiographs. The two raters were well-acquainted with pelvic and coxal structures and radiologic indexes, and measured hip displacement in accordance with standardized methods. ${ }^{4}$

The outermost edge of the acetabular roof was regarded as the datum point from where the Perkin's line (P-line) was drawn. Likewise, another P-line was drawn from the lateral edge of the sourcil. The former and the
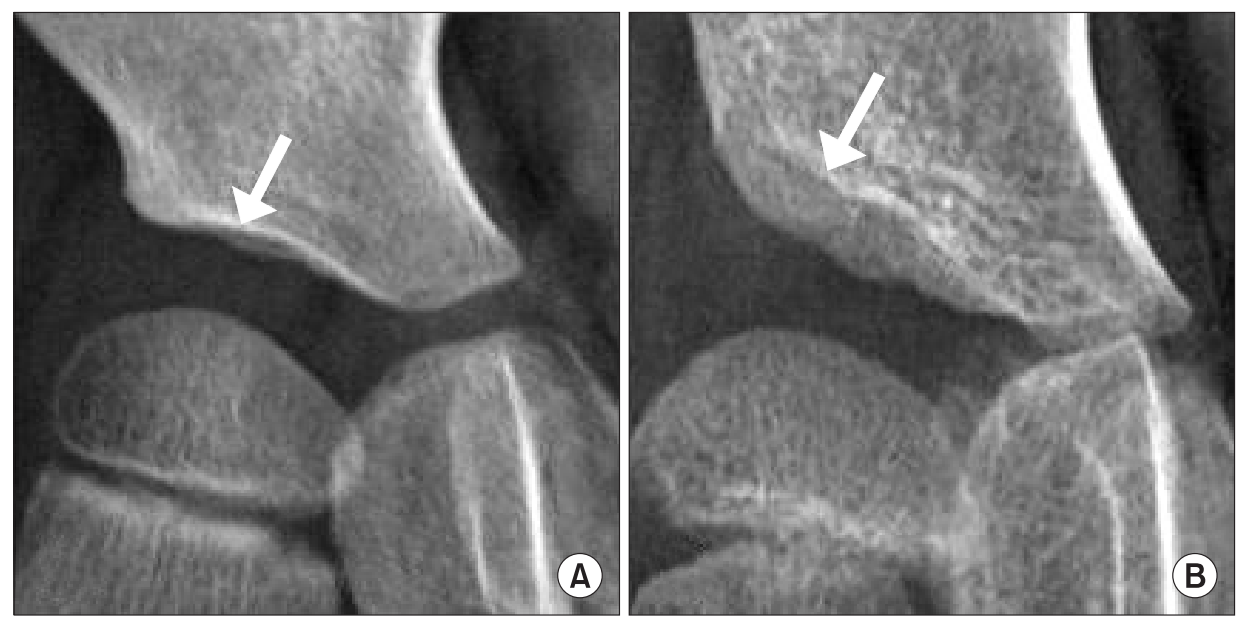

Fig. 1. Sourcil (arrow) in the normal hip (A) and dysplastic hip (B). 
latter method were defined as the classic and modified method, respectively (Fig. 2).

To evaluate intra-rater reliability, the displacement of 200 hip joints was measured 3 times at intervals of 1 week without data on previous measurements, and an analysis was made of the reliability amongst measured values. In order to evaluate inter-rater reliability, the displacement of 200 hip joints was measured in the same way, and an analysis was made of the reliability amongst mean values.

The reliabilities were evaluated in patients with or without hip dysplasia. In accordance with the descriptions of Ogata et al $^{13}$ and Agus et al., ${ }^{8}$ the acetabular roof was classified as to the shape of the sourcil. Type I and II cases where the lateral edge of the acetabular roof and that of the sourcil are superimposed, were regarded as normal. On the other hand, type III and IV were classified as hip dysplasia.

\section{Statistical analysis}

Intra-rater reliability and inter-rater reliability were evaluated by use of Intraclass Correlation Coefficient (ICC), and the standard error of measurement (SEM) was calculated on the basis of inter-value differences and standard deviation. The data were analyzed by use of the program SPSS 18.0.

\section{RESULTS}

Intra-rater reliability was within reliable levels for both raters. In the case of rater-A, ICC indicated 0.94 and 0.90 in the classic method and the modified method, respectively. SEM indicated 8.64 to 11.94 and 12.48 to 17.14 respectively. In the case of rater-B, ICC was 0.97 in both methods. SEM indicated 5.98 to 8.84 and 6.23 to 9.45 , respectively, and thus the error range was narrower in the
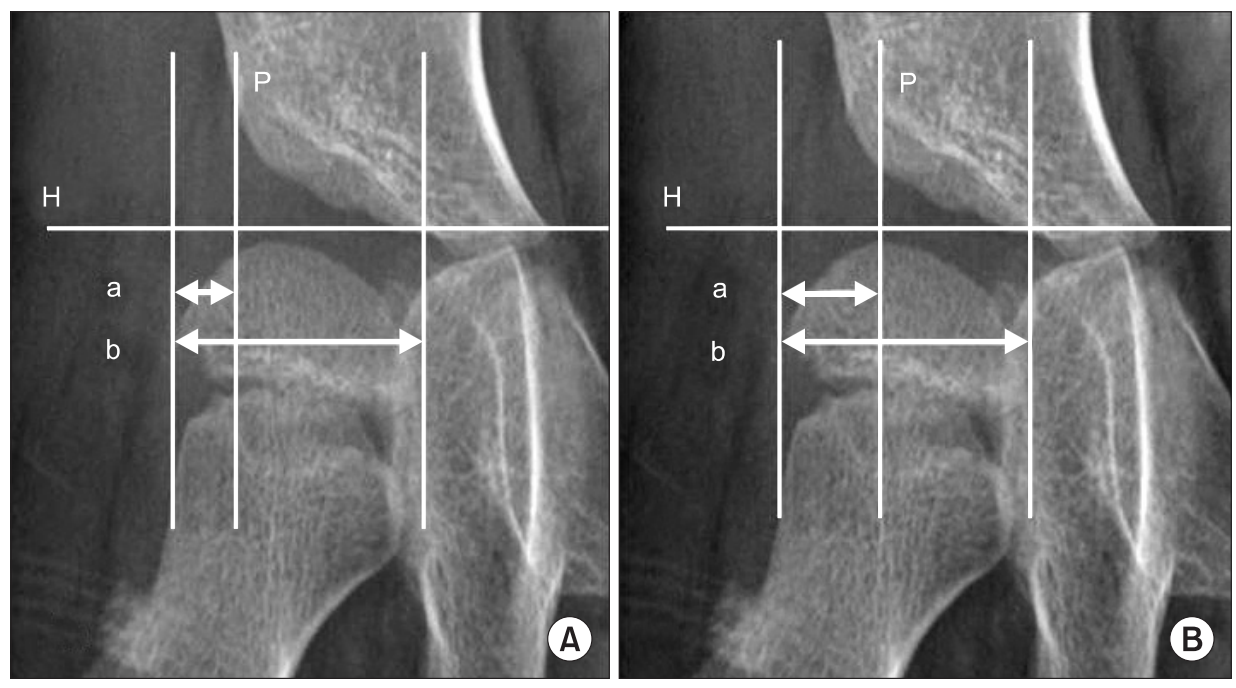

Fig. 2. Migration Index (MI) is measured with the classic method (A) and the modified method (B). H: Hilgenreiner's line, P: Perkin's line, $\mathrm{MI}=\mathrm{a} / \mathrm{b} \times 100(\%)$.

Table 1. Intra-rater and Inter-rater Reliability of Migration Index Measured with Classic and Modified Method

\begin{tabular}{lccr}
\hline & ICC & 95\% confidence interval & SEM \\
\hline $\begin{array}{l}\text { Intrarater reliability of A } \\
\text { Classic method }\end{array}$ & 0.94 & $0.93-0.96$ & $8.65-11.94$ \\
$\quad$ Modified method & 0.90 & $0.88-0.92$ & $12.47-17.14$ \\
$\begin{array}{l}\text { Intrarater reliability of B } \\
\text { Classic method }\end{array}$ & 0.97 & $0.93-0.96$ & $5.98-8.84$ \\
$\quad$ Modified method & 0.97 & $0.93-0.96$ & $6.23-9.45$ \\
Interrater reliability & & & 9.06 \\
$\quad$ Classic method & 0.95 & $0.94-0.96$ & 15.89 \\
$\quad$ Modified method & 0.87 & $0.83-0.90$ & \\
\hline
\end{tabular}

ICC: Intraclass corelation coefficient, SEM: Standard error of measurement 
Sun Mi Kim, et al.

Table 2. Intra-rater and Inter-rater Reliability of Migration Index According to Hip Dysplasia

\begin{tabular}{|c|c|c|c|c|}
\hline & Hip dysplasia & ICC & $95 \%$ confidence interval & SEM \\
\hline \multicolumn{5}{|c|}{ Intrarater reliability of A } \\
\hline \multirow[t]{2}{*}{ Classic method } & Normal hip (n=48) & 0.94 & $0.90-0.96$ & $6.29-10.54$ \\
\hline & Dysplastic hip (n=152) & 0.94 & 0.93-0.96 & $9.40-12.45$ \\
\hline \multirow[t]{2}{*}{ Modified method } & Normal hip (n=48) & 0.89 & $0.84-0.94$ & $8.45-14.80$ \\
\hline & Dysplastic hip (n=152) & 0.89 & $0.86-0.92$ & $12.88-17.95$ \\
\hline \multicolumn{5}{|c|}{ Intrarater reliability of B } \\
\hline \multirow[t]{2}{*}{ Classic method } & Normal hip (n=48) & 0.97 & 0.93-0.96 & $5.24-7.89$ \\
\hline & Dysplastic hip (n=152) & 0.97 & $0.93-0.96$ & $6.10-9.43$ \\
\hline \multirow[t]{2}{*}{ Modified method } & Normal hip (n=48) & 0.96 & $0.92-0.96$ & $5.37-8.32$ \\
\hline & Dysplastic hip (n=152) & 0.95 & $0.91-0.97$ & $6.89-9.90$ \\
\hline \multicolumn{5}{|l|}{ Interrater reliability } \\
\hline \multirow[t]{2}{*}{ Classic method } & Normal hip (n=48) & 0.96 & $0.93-0.96$ & 6.65 \\
\hline & Dysplastic hip (n=152) & 0.95 & $0.93-0.96$ & 9.82 \\
\hline \multirow[t]{2}{*}{ Modified method } & Normal hip (n=48) & 0.95 & $0.91-0.97$ & 7.70 \\
\hline & Dysplastic hip (n=152) & 0.85 & $0.80-0.89$ & 17.89 \\
\hline
\end{tabular}

ICC: Intraclass corelation coefficient, SEM: Standard error of measurement

classic method (Table 1).

In the case of Inter-rater reliability, ICC indicated 0.95 and 0.87 in the classic method and the modified method, respectively. SEM indicated 9.06 and 15.89, respectively, and thus was higher in the classic method (Table 1).

Intra-rater reliability was evaluated in both methods and groups. In the case of rater-A, ICC indicated 0.94 and 0.89 in the classic method and the modified method, respectively, irrespective of group. On the other hand, SEM indicated 8.45 to 14.80 and 12.88 to 17.95 in the normal group and the dysplasia group, respectively, and was thus lower in the normal group. In the case of rater-B, ICC in the classic method indicated 0.97 irrespective of group. In the modified method, it indicated 0.96 and 0.95 in the normal group and the dysplasia group, respectively. SEM was lower in the normal group, which was similar to that of rater-A (Table 2).

Inter-rater reliability was evaluated in both methods and in both groups. In the case of the classic method, ICC indicated 0.96 (SEM: 6.65) and 0.95 (SEM: 9.82) in the normal group and the dysplasia group respectively, and thus it was higher in the normal group. In the case of the modified method, it indicated 0.95 (SEM: 7.70) and 0.85 (SEM: 17.89) respectively. Likewise, it was higher in the normal group (Table 2).

\section{DISCUSSION}

The subluxation or dislocation of the hip joint can be conveniently identified by frontal-view radiographs. For this, various indexes including the migration index, the acetabular index and center-edge angle are widely used. The selection of such indexes should be based on the patient's age or disease. The migration index is to show how much the ossified femoral head has been dislocated from the ossified acetabular roof, which is widely used in children with cerebral palsy who have open triradiate cartilages. ${ }^{4,14}$

The integration index can be easily measured, and its SEM is within $\pm 10 \%$. It can be easily applied to a broad age range and thus may be the most useful index to check for subluxation of the hip joint in children with cerebral palsy. ${ }^{4}$ In many studies, follow-ups were conducted on migration indexes for several months, and as a result the significant progress of hip dysplasia was defined as where SEM was over $10 \%$. In addition, these studies reported that the migration index was useful to evaluate the effects of therapeutic interventions, such as selective dorsal rhizotomy, hip abductors and soft tissue surgery, in the subluxation of the hip joint. ${ }^{15-20}$

A follow-up on the migration index involves the proof of a decrease or increase in hip dysplasia. In this regard, 
it is imperative to secure intra-rater and inter-rater reliabilities. In the study conducted by Parrott et al. ${ }^{5}$ in 2002, 20 hip joints were measured 2 times by 2 raters. Inter-rater and intra-rater reliabilities were high in that ICC was over 0.9 . SEM indicated $\pm 8.3 \%$ in relation to intra-rater reliability. This suggests that when hip migration occurred, SEM reached over $8.3 \%$ when one rater conducted a follow-up. In the study conducted by Pountney et al. ${ }^{6}$ in 2003, 40 hip joints were measured 3 times by 3 raters. SEM indicated $\pm 7.6 \%$ and $\pm 8.1 \%$ in relation to intra-rater reliability and inter-rater reliability respectively, which implies that the reliability was clinically reliable like previous studies.

In the study of Faraj et al. ${ }^{7}$ conducted on 44 hip joints of patients with cerebral palsy (2004), intra-rater reliability was not over $13 \%$ (95\% confidence interval) but interrater reliability reached 21 to $23 \%$, and thus the results were inapplicable clinically. They maintained that the migration index should be measured by one rater.

Error is caused by measurement variability, inaccurate measurements or inaccurate records. In particular, the accurate determination of the datum point is critical for measurement of the migration index. ${ }^{6}$ Nevertheless, the datum point was specified only in the study of Parrott et al. $^{5}$

In this study, the datum point was specified with the classic method and the modified method, whereas intrarater and inter-rater reliabilities showed high levels with the ICC of over 0.9 , which shows that the accurate determination of the datum point raises the reliability.

In relation to intra-rater reliability, SEM was lower in the classic method than in the modified method, which was similar to the results of previous studies.

In the normal hip joint, the sourcil is evenly thick and semicircular. Its lateral part is horizontal or downwardly. ${ }^{9,10}$ In case the sourcil inclines upward or is irregular in thickness, it applies uneven stress to the hip joint. Thus, the correct form of sourcil may be a criterion for acetabular dysplasia (Fig. 1). In 2000, Kim et al. ${ }^{11}$ reported that the inter-rater error may be reduced when the lateral edge of the sourcil was used as the datum point for the measurements of the migration index and the center-edge angle. In addition, they studied the anatomic structure of the sourcil by use of MRI, 3D CT and arthrographies. According to the results, the lateral edge of the sourcil fell under the lateral center of the acetabular roof. However, on radiographs the shadow of the lateral bone meant the anterolateral edge of the acetabular roof, i.e., the inferior region of the anterior inferior iliac spine.

In previous studies, it was reported that the modified method may effectively distinguish dysplastic hip joints from normal ones. ${ }^{8,13}$ However, in this study it was found that the modified method was inferior to the classic method in intra-rater and inter-rater reliabilities. As the case stands, the modified method is likely to produce unreliable results if the migration index is measured by different raters or in cases of severe hip dysplasia.

\section{CONCLUSION}

This study was conducted to compare the classic and modified method in reliability, with children that had cerebral palsy. As mentioned, the former is to use the lateral edge of the acetabular roof as the datum point, and the latter is to use the lateral edge of the sourcil. The results showed that the lateral edge of the acetabular roof was a more effective datum point than that of the sourcil, in terms of reliability. Particularly in relation to hip dysplasia, the lateral edge of the acetabular roof was found to be more reliable as the datum point than that of the sourcil. This proves how important the datum point is in measuring the migration index of children with cerebral palsy. In conclusion, the lateral edge of the acetabular roof may be more effective than that of the sourcil.

\section{REFERENCES}

1. Gamble JG, Rinsky LA, Bleck EE. Established hip dislocation in children with cerebral palsy. Clin Orthop Relat Res1990; 253: 90-99

2. Laplaza FJ, Root L. Femoral anteversion and neckshaft angles in hip instability in cerebral palsy. J Pediatr Orthop 1994; 14: 719-723

3. Hagglund G, Andersson S, Duppe H, Lauge-Pedersen H, Nordmark E, Westbom L. Prevention of dislocation of the hip in children with cerebral palsy. The first ten years of a population-based prevention programme. J Bone joint Surg Br 2005; 87: 95-101

4. Reimers J. The stability of the hip in children. A radiological study of the results of muscle surgery in 
cerebral palsy. Acta Orthop Scand Suppl 1980; 184: $1-100$

5. Parrott J, Boyd RN, Dobson F, Lancaster A, Love S, Oates J, Wolfe R, Nattrass GR, Graham HK. Hip displacement in spastic cerebral palsy: repeatability of radiologic measurement. J Pediatr Orthop 2002; 22: 660-667

6. Pountney T, Mandy A, Gard P. Repeatability and limits of agreement in measurement of hip migration percentage in children with bilateral cerebral palsy. Physiotherapy 2003; 89: 276-281

7. Faraj S, Atherton WG, Stott NS. Inter- and intrameasurer error in the measurement of Reimers' hip migration percentage. J Bone Joint Surg Br 2004; 86: 434-437

8. Agus H, Bicimoqlu A, Omeroglu H, Tumer Y. How should the acetabular angle of Sharp be measured on a pelvic radiograph? J Pediatr Orhop 2002; 22: 228-231

9. Armand M, Lepisto J, Tallroth K, Elias J, Chao E. Outcome of periacetabular osteotomy: joint contact pressure calculation using standing AP radiographs, 12 patients followed for average 2 years. Acta Orthop 2005; 76: 303-313

10. Broughton NS, Brougjman DI, Cole WG. Menelaus MB. Reliability of radiological measurement in the assessment of the child's hip. J Bone Joint Surg Br 1989; 71: 6-8

11. Kim HT, Kim JI, Yoo CI. Diagnosing childhood acetabular dysplasia using the lateral margin of the sourcil. J Pediatr Orthop 2000; 20: 709-717
12. Lonstein JE, Beck K. Hip dislocation and subluxation in cerebral palsy. J Pediatr Orthop 1986; 6: 521-526

13. Ogata S, Moriya H, Tsuchiya K, Akita T, Kamegaya N, Someya M. Acetabular cover in congenital dislocation of the hip. J Bone Joint Surg Br 1990; 72: 190-196

14. Scrutton D. The early management of hips in cerebral palsy. Dev Med Child Neurol 1989; 31:108-116

15. Shea KG, Coleman SS, Carroll K, Stevens P, Van Boerum DH. Pemberton pericapsular osteotomy to treat a dysplastic hip in cerebral palsy. J Bone Joint Surg Am 1997; 79: 1342-1351

16. Cottalorda J, Gautheron V, Metton G, Charmet E, Maatougui K, Chavrier Y. Predicting the outcome of adductor tenotomy. Int Orthop 1998; 22: 374-379

17. Heim RC, Park TS, Vogler GP, Kaufman BA, Noetzel MJ, Ortman MR. Changes in hip migration after selective dorsal rhizotomy for spastic quadriplegia in cerebral palsy. J Neurosurg 1995; 82: 567-571

18. Onimus M, Allamel G, Manzone P, Laurain JM. Prevention of hip dislocation in cerebral palsy by early psoas and adductors tenotomies. J Pediatr Orthop 1991; 11: 432-435

19. Park TS, Vogler GP, Phillips LH, Kaufman BA, Ortman MR, McClure SM, Gaffney PE. Effects of selective dorsal rhizotomy for spastic diplegia on hip migration in cerebral palsy. Pediatr Neurosurg 1994; 20: 43-49

20. Reimers J, Poulsen S. Adductor transfer versus tenotomy for stability of the hip in spastic cerebral palsy. J Pediatr Orthop 1984; 4: 52-54 